\title{
Research on recovery strategy in embedded real-time main memory databases
}

\author{
Tan Yonghong ${ }^{1^{*}}$ and Yin Xiangdong ${ }^{2}$
}

\begin{abstract}
In order to recover data from embedded real-time main memory databases effectively and efficiently, this paper proposes a real-time log-based recovery approach. With respect to the real-time requirement in embedded systems, we classify the consistency in real-time main memory databases into data and transaction consistencies, analyze them theoretically, design rules for correct recovery strategy, and propose real-time log-based recover algorithms for different types of transactions. The experiments show that the proposed approach is more effective and efficient than methods in both traditional and eXtremeDB database systems.
\end{abstract}

Keywords: Embedded system, Real-time main memory database, Recovery strategy, Consistency

\section{Review}

With the development of embedded systems, the application of databases in embedded systems [1] is a hotspot in both industry and academia. Embedded systems work in an environment without manual intervention, so when a fault occurs in these systems, they need to diagnose the fault and recover it automatically all by themselves [2]. The main memory databases $[3,4]$ can reduce the I/O operations greatly while running, and satisfy the real-time requirement of embedded systems, so the databases implemented in embedded systems usually work in the main memory.

In real-time main memory databases [5-7], the main copy of database works in the volatile RAM, and the data is very vulnerable, so the recovery is necessary. Meantime, the I/O operations in real-time main memory databases are few, and recovery is the only part that affects the I/O performance, so the performance of recovery is critical for real-time main memory databases $[8,9]$. While recovering from a fault, real-time main memory databases need to satisfy multiple constraints $[10,11]$, and this pose a huge challenge for designing reasonable recovering strategies.

\footnotetext{
* Correspondence: hunantanyonghong@sina.com

${ }^{1}$ Experimental Training Center, Hunan University of Science and Engineering, YongZhou City, Hunan Province, China

Full list of author information is available at the end of the article
}

Checkpoint or memory snapshots $[12,13]$ is a commonly used program recovery strategy, but the overhead of storing states of running program is very high, and it is not suitable for embedded applications. In addition, the logs in embedded systems record the behaviors of embedded systems, and researchers use different logs to design different recovery strategies, such as partition log [14], real-time $\log$ [15], remote log [16], and operation $\log [17]$. However, these strategies only take the requirement of real-time into consideration, and ignore other specific requirements in embedded systems, so they cannot be applied to the embedded environment efficiently. In addition, the method proposed in [13], studied the recovery strategy in main-memory, but the method is based on virtual memory snapshots. In order to improve real-time ability, Levy and Silberschatz [18] proposed an incremental recovery strategy in mainmemory database.

In this paper, we analyze the consistency constraints in embedded real-time main memory databases from the perspectives of both data and transaction. Then we design some rules that an efficient recovery strategy must obey in embedded real-time main memory databases. Finally, we propose corresponding recovery algorithms for different tasks of embedded real-time main memory databases. 


\section{Analysis of consistency in embedded real-time main memory databases}

In this section, we analyze the consistency of embedded real-time main memory databases from the perspective of both data and transaction.

\subsection{Data consistency}

The embedded real-time main memory databases include three types of data, i.e., image objects, derived objects, and invariant objects.

The objects of real world are sensed by sensors, and their values are written into the databases. The values written into the databases are image objects. An image object is an image of a real world object at some instant, and each image object has its own sampling timestamp and external validity interval.

A derived object is calculated out by a group of image objects during a transaction processing. The timestamp of a derived object is the instant when the transaction is finished, and the validity interval is the intersection of all validity intervals of image objects in the group.

An invariant object is a constant which is invariant as time goes by. The validity of an invariant object is not affected by time, so it is also called non-time series data object.

As there is a validity interval for each image object and derived object, both of them are time series data objects. The sampling time and computing time of time series data are validate only in an interval starting from the system's current time.

Definition 1. If $V I(X)$ is far less than $A T(X)$, i.e., $V I(X)<<A T(X)$, then $X$ is short time-limited data.

The data consistency of embedded real-time systems includes internal consistency, external consistency, and mutual consistency.

Definition 2. $X$ is internal consistent, if and only if it satisfies the predefined integrity and consistency of traditional database systems.

Here, the internal consistency is the internal consistency in traditional database systems, and it only refers to the internal world of database systems.

Definition 3. $X$ is external consistent, if and only if it satisfies $t \leq S T(X)+V I(X)$.

The external consistency requires that the sampling data in a database lag the real world within a certain time.

Definition 4. A group of related data used for decision or deriving new data is a mutual consistent set $R$, and each $R$ is related to a corresponding mutual validity internal $R_{\text {mvi }}$.

Definition 5. Let $R=\left\{X_{1}, X_{2}, \ldots, X_{n}\right\}$, then $R$ is mutual consistent, if and only if $\forall X_{i} \in R, \forall X_{j} \in R$ and $k \neq i$, such that $\left|S T\left(X_{i}\right)-S T\left(X_{j}\right)\right| \leq R_{\mathrm{mvi}}$.
If $R$ is used to generate new data, then the mutual consistency is used to assure the values in $R$ are generated within the common validity interval.

\subsection{Transaction consistency}

The embedded real-time main memory database systems interact with real world according to two behaviors. The first one is recording the states and events of the real world into the databases, and the second one is doing some acts to affect the real world. The embedded real-time transactions can be classified into data receiving transactions, data processing transactions and manipulating transactions.

Data receiving transactions sample the external environment periodically and write it into the databases. This kind of transaction generates an image object in one period, and it is a read-only and non-blocking hard realtime transaction.

Data processing transactions do read-only operations to image objects periodically or nonperiodically, and read and write deriving objects or invariant objects. This kind of transaction does not interact with the real world, and is a soft real-time transaction.

Manipulating transactions read all kinds of data in a database, and do a set of actions $A S(T)=\left\{A_{i} \mid 1 \leq i \leq\right.$ $h$ ) to control the embedded system. If this kind of transaction exceeds the validity interval, disastrous results will be generated, so it is also a hard realtime transaction. Manipulating transactions are readonly operations, and they do not affect the consistency of databases, but they can change the states of real world.

The same as data consistency, transaction consistency in embedded real-time main memory database systems also include internal consistency, external consistency, and mutual consistency.

Definition 6. $T$ is internal consistent, if and only if the value it reads and/or writes satisfies the predefined internal integrity and consistency of traditional database systems.

Definition 7. $T$ is external consistent, if and only if $t \leq D(T)$ and $\forall X_{i} \in D S(T), t \leq S T\left(X_{i}\right)+V I\left(X_{i}\right)$.

The external consistency of embedded real-time transactions requires that each transaction is in its validity internal, and all read/write operations are within its validity interval.

Theorem 1. Let $M V I(T)$ be the minimum of all validate terminal instants of $T$ while reading/writing data objects, then the final terminal instant of $T$ is $D_{R}(T)=\min (D(T)$, $M V I(T))$.

Proof: If $M V I(T)<t<D(T)$, then $\exists X_{i} \in D S(T)$, such that $t>S T\left(X_{i}\right)+\operatorname{VI}\left(X_{i}\right)$, that is, there exists some $X_{i}$, which loses the external consistency, so this violates 
the external consistency constraint while $T$ reads/ writes data objects. On the contrary, if $D(T)<M V I(T)$ and $t>D(T)$, then $T$ exceeds the validity interval, and this violates the external constraint of $T$. So, we can have $D_{R}(T)=\min (D(T), M V I(T))$.

Definition 8. $T$ is mutual consistent, if and only if $\forall X_{i}, X_{j} \in D S(T)$, and $i \neq j$, such that $\left|S T\left(X_{i}\right)-S T\left(X_{j}\right)\right| \leq$ $R_{\text {mvi }}$.

The mutual consistency of embedded real-time transactions means that the time interval between any two data objects is not bigger than the given value $R_{\mathrm{mvi}}(T)$.

With the same reason, when $T$ is both external consistent and mutual consistent, then it is time consistent. A validate submit of transaction in embedded real-time systems depends not only on the internal consistency, but also on the time consistency. So, we have the following corollary.

Corollary 1. $T$ is consistent, if and only if the following constraints satisfy at the same time:

(1) $\forall X_{i}, X_{i} \in D S(T)$;

(2) $C T(T) \leq D_{R}(T)$;

(3) $\forall X_{i} \in R S(T), R T_{T}\left(X_{i}\right) \leq S T\left(X_{i}\right)+V I\left(X_{i}\right)$;

(4) $\forall X_{i}, X_{j} \in R S(T)$ and $i \neq j$, such that $\mid S T\left(X_{i}\right)-$ $S T\left(X_{j}\right) \mid \leq R_{\mathrm{mvi}}(T)$.

\section{Rules for correct recovery strategy}

Taking the internal consistency and time consistency of transactions and data in embedded real-time main memory databases into consideration, we present some rules for correct recovery strategies.

\subsection{Non-time series data recovery rule}

Rule 1. If $T$ has not been submitted, then for $\forall X_{i} \in$ $U S(T)$ satisfying $S_{t}\left(X_{i}\right)=U I_{T}\left(X_{i}\right)$, execute the undo operation.

Rule 2. If $T$ has been submitted, then for $\forall X_{i} \in U S(T)$ satisfying $S_{t}\left(X_{i}\right) \neq U I_{T}\left(X_{i}\right)$, execute the redo operation.

Rules 1 and 2 can recover the data such that they satisfy the internal consistent constraint, and nontime series data only have internal consistent constraint, so they can also be used to recover non-time series data.

\subsection{Time series data recovery rule}

Rule 3. If $\exists X_{i} \in U S(T)$ satisfying $S_{t}\left(X_{i}\right)=U I_{T}\left(X_{i}\right)$ and $t \leq S T\left(X_{i}\right)+V I\left(X_{i}\right)$, then whether or not $T$ has been submitted, there is no need to execute any recovery operation for $X_{i}$.

Rule 4. If $\exists X_{i} \in U S(T)$ satisfying $S_{t}\left(X_{i}\right) \neq U I_{T}\left(X_{i}\right)$ and $t \leq S T\left(X_{i}\right)+V I\left(X_{i}\right)$, then execute the redo operation for $X_{i}$.
Rule 5. If $\exists X_{i} \in U S(T)$ satisfying $t>S T\left(X_{i}\right)+V I\left(X_{i}\right)$, then resample by starting the data receiving transaction of $X_{i}$.

Theorem 2. Rules 3 5 can recover the internal and external state consistency of time series data.

Proof: The recovery of time series data $X_{i}$ needs to consider the consistency between its internal state $S_{t}\left(X_{i}\right)$ with its external state $U I_{T}\left(X_{i}\right)$, but not whether or not the transaction has been submitted.

When $t \leq S T\left(X_{i}\right)+V I\left(X_{i}\right)$, if $S_{t}\left(X_{i}\right) \neq U I_{T}\left(X_{i}\right)$, i.e., the internal and external states of $X_{i}$ are not consistent, then whether or not $T$ has been submitted, the redo operation should be executed according to $U I_{T}\left(X_{i}\right)$ (Rule 4); and if $S_{t}\left(X_{i}\right)=U I_{T}\left(X_{i}\right)$, i.e., the internal and external states of $X_{i}$ are consistent, then whether or not $T$ has been submitted, there is no need to execute any recovery operation (Rule 3 ).

When $t>S T\left(X_{i}\right)+V I\left(X_{i}\right)$, executing undo or redo operation is meaningless, and data receiving transaction should be restarted immediately to resample and recover the consistency of $X_{i}$ between its internal and external states (Rule 5).

\subsection{Real world state recovery rule}

In embedded real-time applications, if the transactions have been submitted and have changed the real world states, there is no need to recover; and if the transactions have not been submitted, then we should do some compensation to recover the state changes of real world.

Rule 6. If $T$ has not been submitted, then for each action that has happened, i.e., $\forall A_{i} \in A S(T)$, execute compensation or recovery task for $A_{i}$.

Theorem 3. Rule 6 can recover the consistency of real world state.

Proof: Manipulating transactions is read-only, and they do not violate the consistency of data objects. The atomicity of manipulating transactions is that, whether all actions of $T, A S(T)=\left\{A_{i} \mid 1 \leq i \leq h\right\}$, are executed or none of them is executed.

Let $O A S(T)=\left\{A_{j} \mid 1 \leq j \leq h\right\}$ be the set of actions that has been executed in $T$ when a fault occurs. According to Rule 6, when $O A S(T) \neq \varnothing$ and $O A S(T) \neq A S(T)$, we need to compensate and recover for $\forall A_{j} \in O A S(T)$. So, the real world states, that have been changed, can be recovered correctly.

\subsection{Transaction restart rule}

No manual intervention is a typical feature of embedded real-time databases, and thus, the database systems should restart all kinds of transactions automatically when faults occur. The transactions needed to restart include two kinds. The first one is that restarting period has passed by or running 
time has exceeded the running period, and the second one includes non-periodic transactions, that do not finish successfully but still satisfy all consistencies.

Rule 7. For a periodic transaction $T$, if $T$ does not finish normally, or $T$ finishes normally and satisfies $t \geq B T(T)+P(T)$, then restart $T$.

Rule 8. For a non-periodic transaction $T$, that does not finish normally, if the following conditions satisfy at the same time, then restart $T$.

(1) $t+E E T(T) \leq D_{R}(T)$

(2) $\forall X_{i} \in R S(T), t \leq S T\left(X_{i}\right)+V I\left(X_{i}\right)$;

(3) $\forall X_{i}, X_{j} \in R S(T), i \neq j$ and $1 \leq i, j \leq n, \mid S T\left(X_{i}\right)-$ $S T\left(X_{j}\right) \mid \leq R_{\mathrm{mvi}}(T)$.

Rule 8 is the same as Corollary 1, i.e., when a fault occurs, only when all consistencies of a transaction have been satisfied, then we can restart the transaction.

\section{Log-based recovery strategy}

In order to recover from faults, embedded real-time main memory databases need to log the time and triggered actions for each transaction and data. These logs include real-time transaction logs, data logs, and action logs. Taking the limits of CPU, storage and energy in embedded systems, we propose the following data recovering strategies based on the rules in the last section.

Strategy 1. If $X$ is a series data with short limited time, then there is no need to log the updates of data.

Strategy 2. If $\frac{\left|A F I\left(X_{i}\right)-B F I\left(X_{i}\right)\right|}{B F I\left(X_{i}\right)} \geq \delta\left(X_{i}\right)$, then log the current data update operation; and otherwise, log nothing.

Strategy 3. Update the time series data objects immediately. That is updating the states of database before a transaction is submitted.

Strategy 4. Deferred update the non-time series data objects. That is updating the states of database when a transaction is submitted.

Strategies 1 and 2 can greatly reduce the overhead of logging the updates of time series data, and also accelerate the recovery speed. Rule 3 makes sure that the latest states of time series data can be written to the databases to reduce the redo operations of time series data. Rule 4 clears the logs of non-time series data and their undo recovery, and can further reduce the overhead of storage and recovery.

Based on the above strategies, we propose corresponding recovery algorithms for data receiving transactions, control transactions, and data processing transactions, and they are described as follows:
(1) Recovery algorithm for data receiving transaction:

Procedure:

1. Scan $T^{\prime} \mathrm{s} \operatorname{logs}$ forward;

2. Determine $U S(T)$;

3. If $U S(T) \neq \varnothing$

4. If $t \leq S T(X)+V I(X)$ then

5. If $S_{t}(X) \neq U I_{T}(X)$ then

6. $\quad$ Let $S_{t}(X) \leftarrow U I_{T}(X)$; //rule 4

$7 . \quad$ Else

8. $\quad$ Restart $T ; / /$ rule 5

9. If $t \geq B T(T)+P(T)$ then

10. Restart $T ; / /$ rule 7

(2) Recovery algorithm for control transactions Procedure:

1. Scan $T^{\prime} \mathrm{s}$ logs forward;

2. Determine $O A S(T)$;

3. If $T$ has not committed then

4. If $O A S(T) \neq \varnothing$ and $O A S(T) \neq A S(T)$ then

5. For $\forall A_{i} \in O A S(T)$ do

6. Do compensating or alternative action of $A$; //rule 6

7. If $t+E E T(T) \leq D_{R}(T)$ and $\forall X \in R S(T)$, $t<S T(X)+V I(X)$ and $\forall X_{i}, X_{j} \in R S(T)$, $\left|S T\left(X_{i}\right)-S T\left(X_{j}\right)\right| \leq R_{m v i}$ then

8. $\quad$ Restart $T ; / /$ rule 8

(3) Recovery algorithm for data processing transactions Procedure:

1. Scan $T^{\prime} \mathrm{s}$ logs forward;

2. Determine the updated temporal data set $U T D S(T)$ and invariant data set $\operatorname{UIDS}(T)$;

3. For $\forall X_{i} \in U T D S(T)$ do

4. If $t<S T(X)+V I(X)$ and $S_{t}\left(X_{i}\right) \neq U I_{T}\left(X_{i}\right)$

5. $\quad$ Let $S_{t}\left(X_{i}\right) \leftarrow U I_{T}\left(X_{i}\right)$; //rule 4

6. For $\forall X_{i} \in U I D S(T)$ do

7. If $T$ has committed then

8. If $S_{t}\left(X_{i}\right) \neq U I_{T}\left(X_{i}\right)$ then

9. Let $S_{t}\left(X_{i}\right) \leftarrow U I_{T}\left(X_{i}\right)$; //rule 2

10. Else $/ / T$ has not committed

11. If $S_{t}\left(X_{i}\right)=U I_{T}\left(X_{i}\right)$ then

12. Let $S_{t}\left(X_{i}\right) \leftarrow B F I\left(X_{i}\right)$; //rule 1

13. $\quad$ Restart $T ; / /$ rule 7

14. If $t>B T(T)+P(T)$ then

15. Restart $T ; / /$ rule 7

\section{Experiments}

\subsection{Experimental setting}

In the experiments, we implement the proposed logbased recovery algorithm on the eXtremeDB embedded database [19], and compare it with the 
traditional recovery method and the method in eXtremeDB. The experiments contain a small database, and the operations include insert, delete, and modification. Query operations are not in our experiments, because they do not change the data in the database, and the recovery strategy does not need to consider this situation. We mainly compare the system overhead, overtime transaction ratio (ratio of transactions that exceed the validity interval), and rejecting service time (downtime). The meanings and values of experimental parameters in eXtremeDB are in Table 1.

\subsection{Experimental results}

Firstly, we compare the CPU utilization and log buffer utilization of the three approaches, and the results are in Figs. 1 and 2, respectively. With respect to CPU utilization, our proposed approach is higher than the other two, and the reason is that the proposed approach uses main memory to store data and it has the highest throughput. With respect to the log buffer utilization, the value of the proposed approach is the lowest, which means that the proposed method only logs necessary data and the usage of log buffer is the most efficient.

Secondly, we compare the ratio of transactions exceeding the validity interval in Fig. 3, and the average rejecting service time in Fig. 4. The ratio of transactions exceeding the validity interval is also the ratio of missing transactions. From Fig. 3, we can see that our proposed approach has the least missing transactions. Rejecting service time is also called downtime. Figure 4 illustrates that the proposed approach has the lowest average downtime.

Next, in our proposed approach, we observe the changes of overtime transaction ratio under different "per_short" (short time-limited data ratio) and

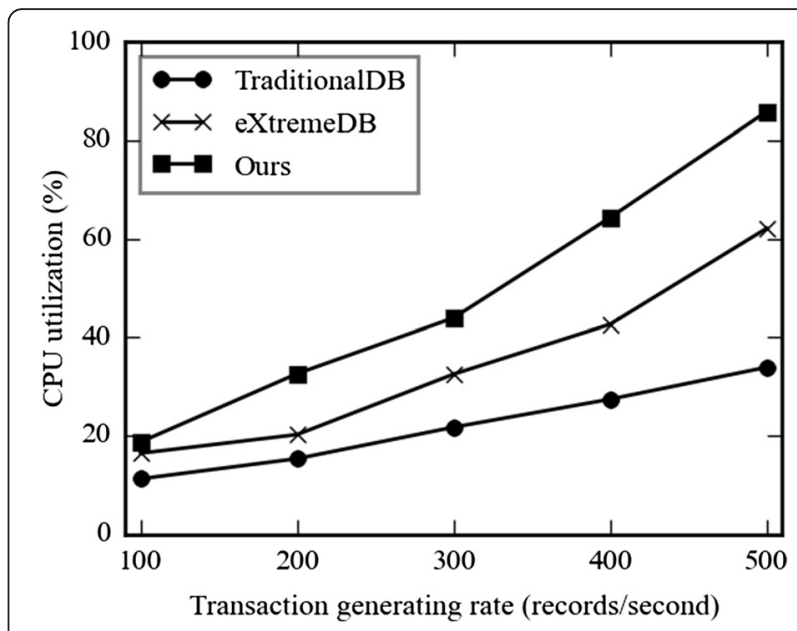

Fig. 1 Comparison of CPU utilization

"threshold" (time series data state change threshold), and the results are in Figs. 5 and 6, respectively. In Fig. 5, the order of overtime transaction ratios for different per_short is $0>0.5>0.1>0.3>0.2$, which means that we must carefully select per_short to optimize the overtime transaction ratio. Here, per_short $=0.2$ is the best. In Fig. 6, the order of overtime transaction ratios for different threshold is the same as that of per_short, so we can have the same conclusion.

Finally, we observe the time series data ratio of the proposed approach under different update modes, and the results are in Fig. 7. From the figure, we can see that the hybrid of deferred and immediate update modes has the lowest time series data ratio, which means that the hybrid update mode has canceled the overhead of undo recovery for the invariant data objects, and thus reduces the ratio of transactions exceeding the validity interval.

Table 1 Parameter setting

\begin{tabular}{|c|c|c|c|}
\hline Parameter & Meaning & Default value & Domain \\
\hline num_imo & Image data object number & 250 & $50 \sim 500$ \\
\hline num_deo & Derived data object number & 250 & $50 \sim 500$ \\
\hline num_ino & Invariant data object number & 500 & $200 \sim 1000$ \\
\hline per_short & Short time limited data ratio & $20 \%$ & $0 \sim 40 \%$ \\
\hline$v i$ & Time series data validate time & $50 \mathrm{~ms}$ & $5 \mathrm{~ms} \sim 10 \mathrm{~s}$ \\
\hline$\delta$ & Time series data state change threshold & $10 \%$ & $0 \sim 20 \%$ \\
\hline period & Period of periodic transaction & $50 \mathrm{~ms}$ & $5 \mathrm{~ms} \sim 10 \mathrm{~s}$ \\
\hline generation_ratio & Periodic transaction generating ratio & $20 / s$ & $5 \sim 50 / s$ \\
\hline trigger_ratio & MT trigger ratio & $5 / \mathrm{s}$ & $2 \sim 10 / s$ \\
\hline update_number & Update number & 2 & $1 \sim 3$ \\
\hline num_actions & Action number & 3 & $1 \sim 5$ \\
\hline update_mode & Update mode & Hybrid & Deferred/immediate/hybrid \\
\hline
\end{tabular}




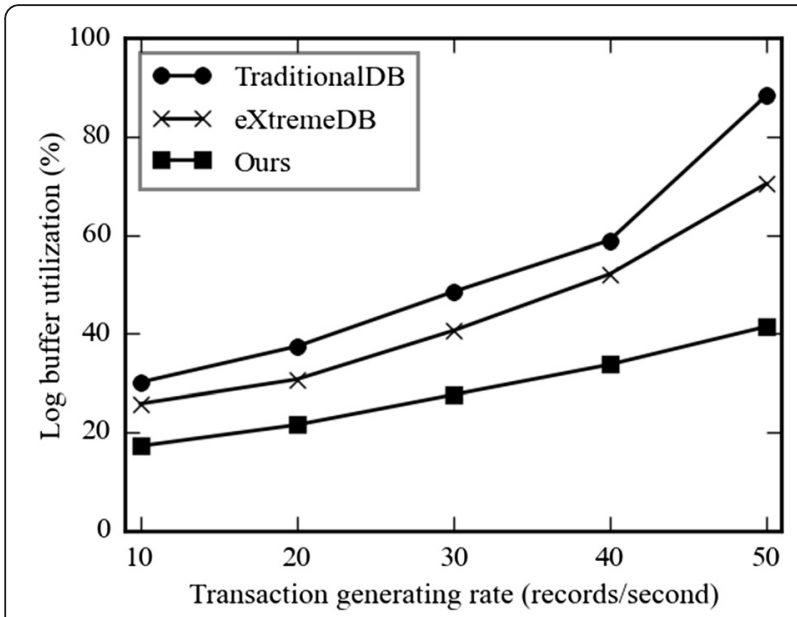

Fig. 2 Comparison of log buffer utilization

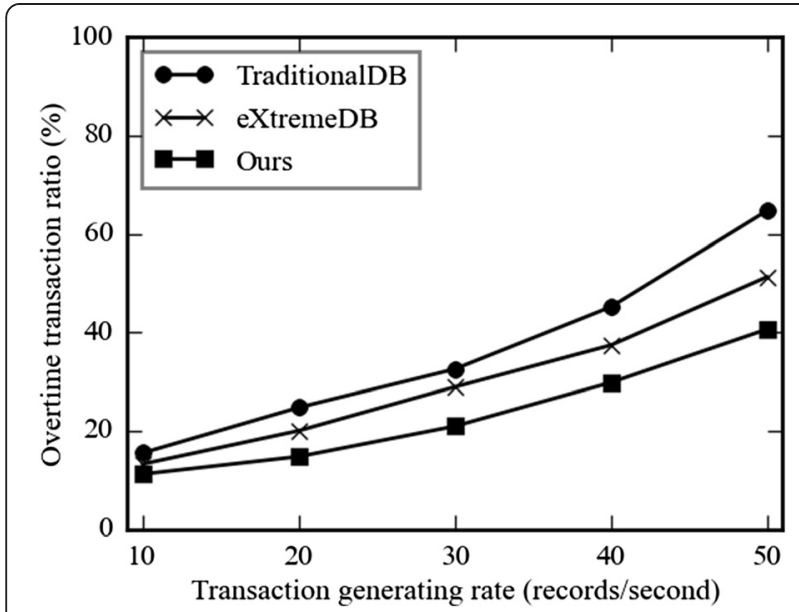

Fig. 3 Comparison of overtime transaction ratio

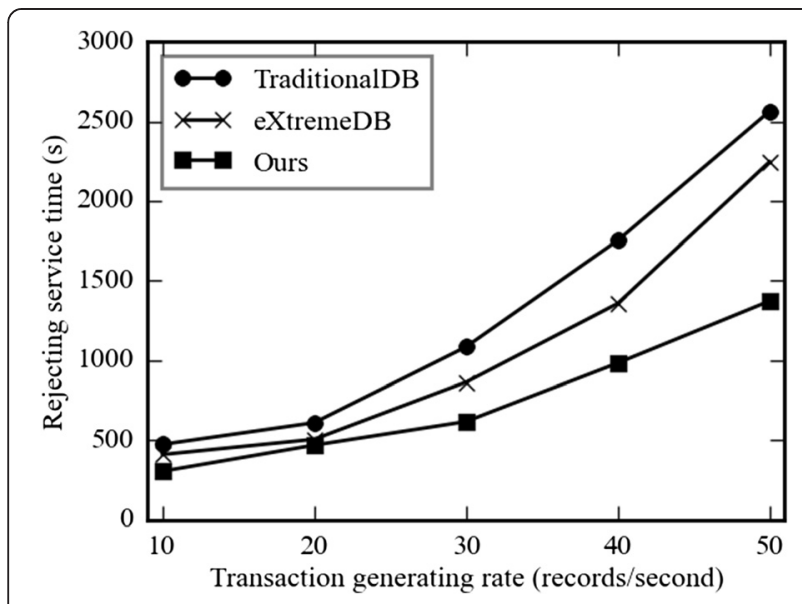

Fig. 4 Comparison of rejecting service time

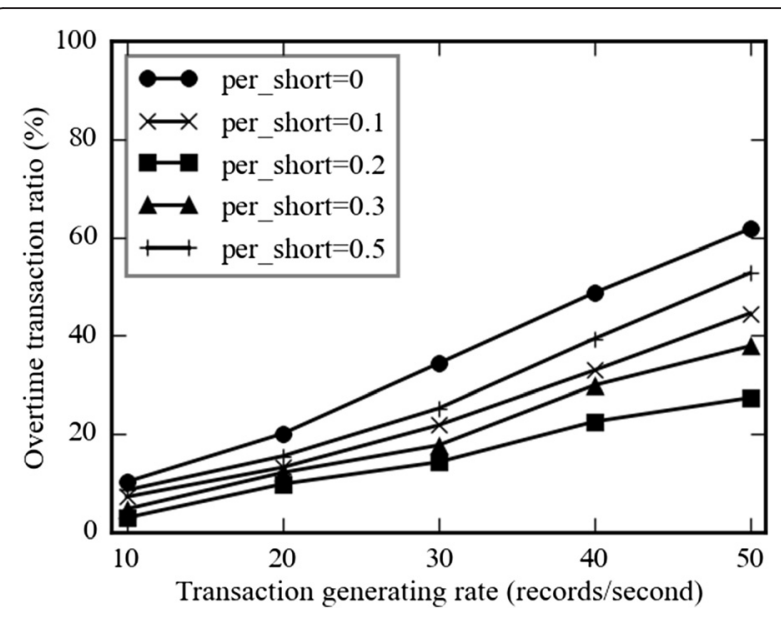

Fig. 5 Overtime transaction ratios for different "per_short"

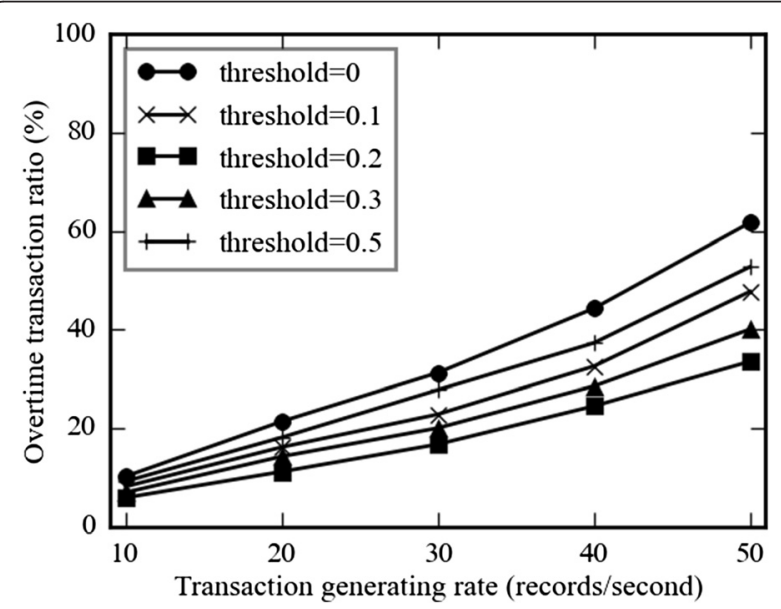

Fig. 6 Overtime transaction ratios for different "threshold"

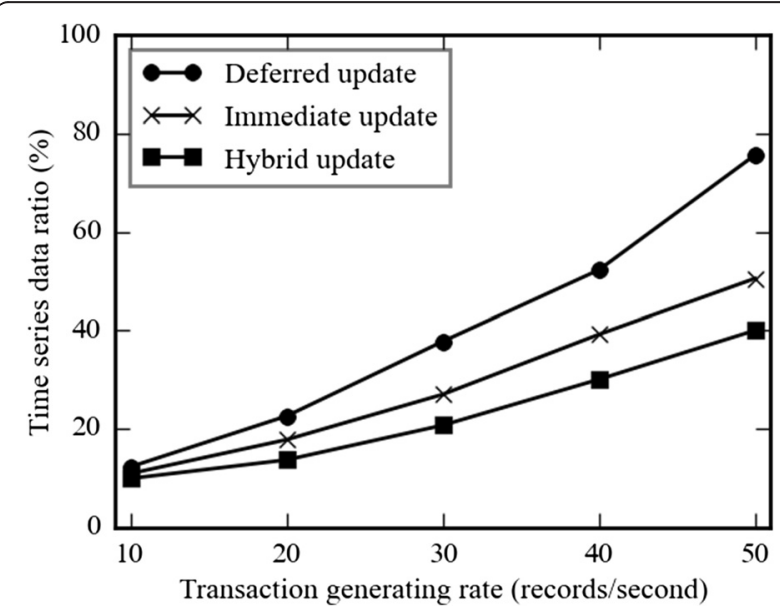

Fig. 7 Time series data ratio for different update modes 


\section{Conclusions}

In this paper, we study the problem of data recovery strategy in embedded real-time main memory databases. Because of real-time requirement in embedded systems, consistency of embedded real-time main memory databases is different from traditional databases. We analyzed both the data and transaction consistencies in embedded real-time main memory databases, designed rules for correct recovery strategy, and proposed real-time log-based recover algorithms for different types of transactions. The experiments show that the proposed approach is more effective and efficient than methods in both traditional and eXtremeDB database systems. The proposed recovery algorithm can be integrated into the eXtremeDB database, and thus provide better recovery performance. Integrating the proposed algorithm into other main memory database will be our future work.

\section{Competing interests}

The authors declare that they have no competing interests.

\section{Acknowledgements}

The work was supported by the following funds: Hunan Provincial Natural Science Foundation of China (Grant No.2015JJ6043); Hunan University of Science and Engineering; Scientific Research Fund of Hunan Provincial Education Department(Grant No.12A054); The Construct Program of the Key Discipline in Hunan University of Science and Engineering(Circuits and Systems).

\section{Author details}

${ }^{1}$ Experimental Training Center, Hunan University of Science and Engineering, YongZhou City, Hunan Province, China. ${ }^{2}$ School of Electronics and Information Engineering, Hunan University of Science and Engineering, YongZhou City, Hunan Province, China.

Received: 22 December 2015 Accepted: 20 April 2016

Published online: 04 May 2016

\section{References}

1. A Nori, Mobile and Embedded Databases[C]//Proceedings of the 2007 ACM SIGMOD International Conference on Management of Data. ACM, 2007, pp. 1175-1177

2. V Narayanan, Y Xie, Reliability concerns in embedded system designs. Computer 39(1), 118-120 (2006)

3. H Garcia-Molina, K Salem, Main memory database systems: an overview. Knowl. Data Eng. IEEE Trans. 4(6), 509-516 (1992)

4. J Stankovic, SH Son, J Hansson, Misconceptions about real-time databases. Computer 32(6), 29-36 (1999)

5. K Ramamritham, Real-time databases. Distrib. Parallel Databases 1(2) 199-226 (1993)

6. G Özsoyoğlu, RT Snodgrass, Temporal and real-time databases: a survey. Knowl. Data Eng. IEEE Trans. 7(4), 513-532 (1995)

7. K Ramamritham, SH Son, LC Dipippo, Real-time databases and data services. Real-time Syst. 28(2-3), 179-215 (2004)

8. KH Kim, HO Welch, Distributed execution of recovery blocks: an approach for uniform treatment of hardware and software faults in real-time applications. Comput. IEEE Trans. 38(5), 626-636 (1989)

9. RM Sivasankaran, K Ramamritham, JA Stankovic et al., Data Placement, Logging and Recovery in Real-Time Active Databases[M]//Active and Real-Time Database Systems (ARTDB-95) (Springer, London, 1996), pp. 226-241

10. Soparkar NR, Silberschatz A, Korth HF. Time-constrained transaction management: real-time constraints in database transaction systems. Kluwer Academic Publishers; 1996.

11. MI Seltzer, MA Olson, Challenges in Embedded Database System Administration[C]//Proceeding of the Embedded System Workshop, 1999, pp. 29-31
12. GM Liao, JP Li, Research on Timely Recovery Technology of Memory Database[C]//Wavelet Active Media Technology and Information Processing (ICWAMTIP), 2012 International Conference on. IEEE, 2012, pp. 268-271

13. A Kemper, T Neumann, HyPer: A hybrid OLTP\&OLAP Main Memory Database System Based on Virtual Memory Snapshots[C]//Data Engineering (ICDE), 2011 IEEE 27th International Conference on. IEEE, 2011, pp. 195-206

14. Lam KY, Kuo TW. real-time database systems: architecture and techniques. Kluwer Academic Publishers; 2001.

15. LC Shu, JA Stankovic, SH Son, Achieving bounded and predictable recovery using real-time logging. Comput. J. 47(3), 373-394 (2004)

16. T Niklander, K Raatikainen, Using Logs to Increase Availability in Real-Time Main-Memory Database[M]//Parallel and Distributed Processing (Springer, Berlin Heidelberg, 2000), pp. 720-726

17. N Malviya, A Weisberg, S Madden et al., Rethinking Main Memory OLTP Recovery[C]//Data Engineering (ICDE), 2014 IEEE 30th International Conference on. IEEE, 2014, pp. 604-615

18. E Levy, A Silberschatz, Incremental recovery in main memory database systems. Knowl. Data Eng. IEEE Trans. 4(6), 529-540 (1992)

19. MC Majhi, AK Behera, NM Kulshreshtha et al., ExtremeDB: a unified web repository of extremophilic archaea and bacteria [J], 2013

\section{Submit your manuscript to a SpringerOpen ${ }^{\circ}$ journal and benefit from:}

- Convenient online submission

- Rigorous peer review

- Immediate publication on acceptance

- Open access: articles freely available online

- High visibility within the field

- Retaining the copyright to your article

Submit your next manuscript at $>$ springeropen.com 